\title{
Uniform asymptotics for the tail probability of weighted sums with heavy tails
}

\author{
Chenhua Zhang ${ }^{1}$
}

\begin{abstract}
This paper studies the tail probability of weighted sums of the form $\sum_{i=1}^{n} c_{i} X_{i}$, where random variables $X_{i}$ 's are either independent or pairwise quasi-asymptotical independent with heavy tails. Using $h$-insensitive function, the uniform asymptotic equivalence of the tail probabilities of $\sum_{i=1}^{n} c_{i} X_{i}, \max _{1 \leq k \leq n} \sum_{i=1}^{k} c_{i} X_{i}$ and $\sum_{i=1}^{n} c_{i} X_{i}^{+}$is established, where $X_{i}$ 's are independent and follow the long-tailed distribution, and $c_{i}$ 's take value in a broad interval. Some further uniform asymptotic results for the weighted sums of $X_{i}$ 's with dominated varying tails are obtained. An application to the ruin probability in a discrete-time insurance risk model is presented.
\end{abstract}

MSC: 41A60; 62P05; 62E20; 91B30

Keywords: $h$-insensitive function, long-tailed distribution, consistently varying tail, dominated variation, quasi-asymptotical independence

\section{Introduction}

In this paper, all asymptotic and limit relations are taken as $x \rightarrow \infty$ unless otherwise stated. For independently and identically distributed (iid) subexponential random variables $X_{i}, i \geq 1$, it is well-known that, for any $n \geq 2$,

$$
P\left(\sum_{i=1}^{n} X_{i}>x\right) \sim P\left(\max _{1 \leq k \leq n} \sum_{i=1}^{k} X_{i}>x\right) \sim P\left(\sum_{i=1}^{n} X_{i}^{+}>x\right) \sim \sum_{i=1}^{n} P\left(X_{i}>x\right),
$$

where $x^{+}=\max \{x, 0\}$. There are quite a few ways to generalize these asymptotic relations. One way is to consider some broader classes of heavy-tailed distributions, see, e.g., Ng et al. [18]. Another way is to study the randomly stopped sums, see, e.g., Denisov et al. [6]. Allowing some dependence of $X_{i}$ 's, similar results can be obtained for different classes of heavy-tailed distributions, see Wang and Tang [22], Geluk and Ng [11], Tang [20], Geluk and Tang [12], and references therein.

A more general way is to work on the weighted sums of form $\sum_{i=1}^{n} c_{i} X_{i}$, where weights $c_{i}$ 's are real numbers. If $X_{i}$ 's are iid subexponential random variables, Tang and Tsitsiashvili [21] proved that for any $0<a \leq b<\infty$, the asymptotic relation

$$
P\left(\sum_{i=1}^{n} c_{i} X_{i}>x\right) \sim \sum_{i=1}^{n} P\left(c_{i} X_{i}>x\right)
$$

holds uniformly for $a \leq c_{i} \leq b, 1 \leq i \leq n$, in the sense that

$$
\lim _{x \rightarrow \infty} \sup _{a \leq c_{i} \leq b, 1 \leq i \leq n}\left|\frac{P\left(\sum_{i=1}^{n} c_{i} X_{i}>x\right)}{\sum_{i=1}^{n} P\left(c_{i} X_{i}>x\right)}-1\right|=0 .
$$

\footnotetext{
${ }^{1}$ Department of Mathematics, The University of Southern Mississippi, Hattiesburg, MS 39406-5045, USA, chenhua.zhang@usm.edu
} 
Recently, Liu et al. [16] and $\mathrm{Li}$ [14] established the same asymptotic relation for some dependent $X_{i}$ 's.

Chen et al. [3] showed that for any fixed $0<a \leq b<\infty$ it holds that uniformly for $a \leq c_{i} \leq b$, $1 \leq i \leq n$,

$$
P\left(\sum_{i=1}^{n} c_{i} X_{i}>x\right) \sim P\left(\max _{1 \leq k \leq n} \sum_{i=1}^{k} c_{i} X_{i}>x\right) \sim P\left(\sum_{i=1}^{n} c_{i} X_{i}^{+}>x\right)
$$

where $X_{i}$ 's are independent, not necessarily identically distributed, random variables with longtailed distributions. This result is extended by substituting $b$ with any positive function $b(x)$ such that $h(x) \nearrow \infty$ and $b(x)=o(x)$ in this paper.

Replacing the constant weights $c_{i}$ 's with random weights $\theta_{i}$ 's, the asymptotic relation (2) and (3) still hold if the weights $\theta_{i}$ 's, independent of $X_{i}$ 's, are uniformly bounded away from zero and infinity. Then it is very natural to consider the randomly weighted sum of form $\sum_{i=1}^{n} \theta_{i} X_{i}$. Wang and Tang [23] obtained $P\left(\sum_{i=1}^{n} \theta_{i} X_{i}>x\right) \sim P\left(\max _{1 \leq k \leq n} \sum_{i=1}^{k} \theta_{i} X_{i}>x\right) \sim P\left(\sum_{i=1}^{n} \theta_{i} X_{i}^{+}>x\right)$ for the case that the random weights are not necessarily bounded and $X_{i}$ 's are independently random variables with common distribution belonging to a smaller class than the class of subexponential distributions. Furthermore, Zhang et al. [24], Chen and Yuen [4] established the same results for dependent $X_{i}$ 's, where the dependence structures of $X_{i}$ 's are essentially same for proof of their results.

The rest of this paper is organized as follows. Section 2 reviews some important classes of heavytailed distributions. Section 3 states the main results along with some corollaries. Section 4 gives an application of the main results to the ruin probability in a discrete-time insurance risk model. The proof of the main results and some lemmas are presented in Section 5.

\section{Classes of Heavy-Tailed Distributions}

A random variable $X$ or its distribution $F$ is said to be heavy-tailed to the right or have a heavy (right) tail if the corresponding moment generate function does not exist on the positive real line, i.e., $E e^{t X}=\int_{-\infty}^{\infty} e^{t x} d F(x)=\infty$ for any $t>0$. The most important class of heavy-tailed distributions is the class of subexponential distributions, denoted by $\mathcal{S}$. Write the tail distribution by $\bar{F}(x)=1-F(x)$ for any distribution $F$. Let $F^{* n}$ denote the $n$-fold convolution of $F$. A distribution $F$ concentrated on $[0, \infty)$ is subexponential if

$$
\overline{F^{* n}}(x) \sim n \bar{F}(x)
$$

for some or, equivalently, for all $n \geq 2$. More generally, a distribution $F$ on $(-\infty, \infty)$ belongs to the subexponential class if $F^{+}(x)=F(x) I_{\{x \geq 0\}}$ does.

Closely related to the subexponential class $\mathcal{S}$, the class $\mathcal{D}$ of dominated varying distributions consists of distributions satisfying

$$
\limsup _{x \rightarrow \infty} \frac{\bar{F}(y x)}{\bar{F}(x)}<\infty
$$


for some or, equivalently, for all $0<y<1$. A slightly smaller class of $\mathcal{D}$ is the class of distributions with consistently varying tail, denoted by $\mathcal{C}$. Say that a distribution $F$ belongs to the class $\mathcal{C}$ if

$$
\lim _{y \searrow 1} \liminf _{x \rightarrow \infty} \frac{\bar{F}(y x)}{\bar{F}(x)}=1 \text { or, equivalently, } \lim _{y \nearrow_{1}} \limsup _{x \rightarrow \infty} \frac{\bar{F}(y x)}{\bar{F}(x)}=1 .
$$

A distribution $F$ belongs to the class $\mathcal{L}$ of long-tailed distributions if

$$
\lim _{x \rightarrow \infty} \frac{\bar{F}(x+y)}{\bar{F}(x)}=1
$$

for some or, equivalently, for all $y$. A tail distribution $\bar{F}$ is called $h$-insensitive if $\bar{F}(x+y) \sim \bar{F}(x)$ holds uniformly for all $|y| \leq h(x)$, where $h(x)$ is a positive nondecreasing function and $\lim _{x \rightarrow \infty} h(x)=\infty$. The concept of $h$-insensitive function is extensively used in the monograph of Foss et al. [9]. For any distribution $F \in \mathcal{L}$, it can be shown that $\bar{F}$ is $h$-insensitive for some positive nondecreasing function $h(x):=h_{F}(x)$ such that $h(x) \nearrow \infty$ and $h(x)=o(x)$, see, e.g., Lemma 5.1 in Section 5 , Section 2 in Foss and Zachary [10], Lemma 4.1 of Li et al. [15]. Consequently, $\bar{F}$ is $c h$-insensitive for any fixed positive real number $c$.

It is known that the proper inclusion relations

$$
\mathcal{C} \subset \mathcal{D} \cap \mathcal{L} \subset \mathcal{S} \subset \mathcal{L}
$$

hold, see, e.g., Embrechts et al. [8], Foss et al. [9].

\section{Main Results}

Throughout the rest of this paper $X_{i}, i \geq 1$, are random variables with distribution $F_{i}, i \geq 1$, respectively. Adopt the notation $M_{c} F$ and $*_{1 \leq i \leq n} M_{c_{i}} F_{i}$ in Barbe and McCormick [1]. For $X \sim F$ and $c>0$, let $M_{c} F(x)=F(x / c)$ be the distribution of $c X$. The distribution of $\sum_{i=1}^{n} c_{i} X_{i}$ is $*_{1 \leq i \leq n} M_{c_{i}} F_{i}$, where $X_{i}, 1 \leq i \leq n$, are independent random variables and $*_{1 \leq i \leq n} M_{c_{i}} F_{i}$ is the convolution of $M_{c_{i}} F_{i}, 1 \leq i \leq n$.

The first main result generalizes Lemma 4.1 of Chen et al. [3] with different approach in two ways. First, it increases the upper bound of the weights and decreases the lower bound of the weights. Second, the fixed shift term $A$ in Lemma 4.1 of Chen et al. [3] is enlarged to some unbounded function, which is irrespective of the upper bound of the weights.

Theorem 3.1. If $X_{i} \sim F_{i} \in \mathcal{L}, 1 \leq i \leq n$, are independent random variables, there exists a positive nondecreasing function $h(x):=h\left(x ; F_{1}, \cdots, F_{n}\right)$ satisfying $h(x) \nearrow \infty$ such that ${ }^{*}{ }_{1 \leq i \leq n} M_{c_{i}} F_{i}$ is uniformly $h(x)$-long-tailed for $a(x) \leq c_{i} \leq b(x), 1 \leq i \leq n$, in the sense that

$$
P\left(\sum_{i=1}^{n} c_{i} X_{i}>x \pm h(x)\right) \sim P\left(\sum_{i=1}^{n} c_{i} X_{i}>x\right)
$$

holds uniformly for $a(x) \leq c_{i} \leq b(x), 1 \leq i \leq n$, i.e.,

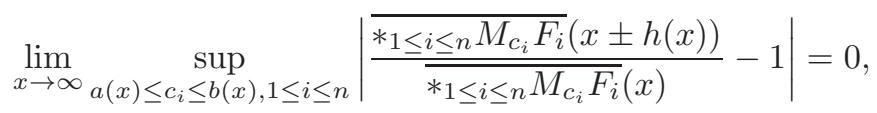

where the positive function $b(x)$ satisfies $b(x) \nearrow \infty$ and $b(x)=o(x), h(x)$ is irrespective of $b(x)$, $a(x)=h^{-\delta}(x) \searrow 0$ for some $\delta>0$. 
Remark 3.1. Considering the case of Weibull distribution $F_{1}(x)=1-e^{-c x^{\tau}} \in \mathcal{S} \subset \mathcal{L}$ with $0<\tau<1$, it indicates that the restriction on $a(x)$ can not be weakened in general.

It is known that the class $\mathcal{L}$ is closed under convolution (see, e.g., Theorem 3 of Embrechts and Goldie [7], Corollary 2.42 of Foss et al. [9]), which can be also derived directly from Theorem 3.1.

Corollary 3.1. If $X_{i} \sim F_{i} \in \mathcal{L}, 1 \leq i \leq n$, are independent random variables, then the distribution of $\left.\sum_{i=1}^{n} c_{i} X_{i}>x\right)$ is long-tailed for any fixed $c_{i}>0,1 \leq i \leq n$. Consequently, the class $\mathcal{L}$ of long-tailed distributions is closed under convolution.

Theorem 3.2. If $X_{i} \sim F_{i} \in \mathcal{L}, 1 \leq i \leq n$, are independent random variables, there exist positive functions $a(x)$ and $b(x)$ satisfying $a(x) \searrow 0$ and $b(x) \nearrow \infty$ such that the asymptotic relations (3) hold uniformly for $a(x) \leq c_{i} \leq b(x), 1 \leq i \leq n$.

The following result can be also founded in Lemma 3.4 of Foss et al. [9].

Corollary 3.2. A distribution $F \in \mathcal{S}$ iff $F \in \mathcal{L}$ and $\overline{F * F}(x) \sim 2 \bar{F}(x)$.

Random variables $X_{i}, i \geq 1$, are pairwise strong quasi-asymptotically independent (pSQAI) if, for any $i \neq j$,

$$
\lim _{\min \left\{x_{i}, x_{j}\right\} \rightarrow \infty} P\left(\left|X_{i}\right|>x_{i} \mid X_{j}>x_{j}\right)=0,
$$

which was used in Geluk and Tang [12], Liu et al. [16] and Li [14], and related to what is called asymptotic independence; see e.g. Resnick [17].

Theorem 3.3. If $X_{i} \sim F_{i} \in \mathcal{C}, 1 \leq i \leq n$, are $p S Q A I$ random variables and $b(x)$ is an arbitrary fixed positive function satisfying $b(x) \nearrow \infty$ and $b(x)=o(x)$, then it holds that, uniformly for any $0<c_{i} \leq b(x), 1 \leq i \leq n$,

$$
P\left(\sum_{i=1}^{n} c_{i} X_{i}>x\right) \sim P\left(\max _{1 \leq k \leq n} \sum_{i=1}^{k} c_{i} X_{i}>x\right) \sim P\left(\sum_{i=1}^{n} c_{i} X_{i}^{+}>x\right) \sim \sum_{i=1}^{n} P\left(c_{i} X_{i}>x\right) .
$$

Corollary 3.3. Under assumption of Theorem 3.3, the above result still holds for $0 \leq c_{i} \leq b(x), 1 \leq$ $i \leq n$, and $\min _{1 \leq i \leq n} c_{i}>0$.

The next theorem extends Lemma 2.1 of Liu et al [16] and Theorem 2.1 of Li [14] with a different proof, which is based on Theorem 3.1.

Theorem 3.4. If $X_{i} \sim F_{i} \in \mathcal{D} \cap \mathcal{L}, 1 \leq i \leq n$, are $p S Q A I$ random variables, there exist a positive function $a(x) \searrow 0$ and a positive function $b(x) \nearrow \infty$ such that (5) holds uniformly for $a(x) \leq c_{i} \leq$ $b(x), 1 \leq i \leq n$.

Remark 3.2. Both $a(x)$ and $b(x)$ depend on $h(x)$ in Theorem 3.2 and 3.4, where $h(x)=o(x)$ is given in Theorem 3.1. More specifically, $a(x)=h^{-\delta}(x)$ for some $\delta>0$ and $b(x)=o(h(x))$, for example, $b(x)=h^{1 / 2}(x)$.

Remark 3.3. If the constant weights $c_{i}, 1 \leq i \leq n$ are replaced by random weights $\theta_{i}, 1 \leq i \leq n$, which are independent of $X_{i}, 1 \leq i \leq n$, conditioning on the random weights can easily establish the corresponding results for random weights sums. 
The proof of Theorem 3.4 gives an extension of Lemma 4.3 of Geluk and Tang [12].

Corollary 3.4. If $X_{i} \sim F_{i} \in \mathcal{L}, 1 \leq i \leq n$, are $p Q S A I$ random variables, it holds that, for some the positive functions $b(x) \nearrow \infty$ and $a(x) \searrow 0$,

$$
\lim _{x \rightarrow \infty} \inf _{a(x) \leq c_{i} \leq b(x), 1 \leq i \leq n} \frac{P\left(\sum_{i=1}^{n} c_{i} X_{i}>x\right)}{\sum_{i=1}^{n} P\left(c_{i} X_{i}>x\right)} \geq 1 .
$$

\section{Application to Risk Theory}

Consider the following discrete-time insurance risk model

$$
U_{0}=x, U_{n}=U_{n-1}\left(1+r_{n}\right)-X_{n}, n \geq 1,
$$

where $U_{n}$ stands an insurer's surplus at the end of period $n$ with a deterministic initial surplus $x, r_{n}$ represents the constant interest force of an insurer's risk-free investment, and the net loss $X_{n}$ over period $n$ equals the total amount of claims plus other costs minus the total amount of premiums during period $n$. It is an interesting and important problem arising from the above discrete-time insurance risk model to study the ruin probabilities of the insurer. See Tang [19] for detailed discussion.

The ruin probability by time $n$ is defined as

$$
\psi(x ; n)=P\left(\min _{i=1}^{n} U_{i}<0 \mid U_{0}=x\right) .
$$

It is easy to see that the surplus process is of form

$$
U_{0}=x, U_{n}=\prod_{i=1}^{n}\left(1+r_{i}\right) x-\sum_{i=1}^{n}\left(\prod_{j=i+1}^{n}\left(1+r_{j}\right)\right) X_{i}, n \geq 1 .
$$

Define the discounted surplus process as follows

$$
\widetilde{U}_{n}=\left(\prod_{i=1}^{n}\left(1+r_{i}\right)\right)^{-1} U_{n}=x-\sum_{i=1}^{n} c_{i} X_{i}
$$

where $c_{i}=\prod_{j=1}^{i}\left(1+r_{j}\right)^{-1}$ represents the discount factor from time $i$ to time $0,1 \leq i \leq n$. Then the corresponding ruin probability can be written as

$$
\psi(x ; n)=P\left(\min _{i=1}^{n} \widetilde{U}_{i}<0 \mid \widetilde{U}_{0}=x\right)=P\left(\max _{1 \leq i \leq k} \sum_{i=1}^{k} c_{i} X_{i}>x\right) .
$$

Applying Theorem 3.2 and Theorem 3.4 in Section 3, the following asymptotic results can be obtained.

Corollary 4.1. Assume that net losses $X_{i}, i \geq 1$ are independent random variables, which are not necessarily identically distributed, with distribution $F_{i}, i \geq 1$, respectively. If $F_{i} \in \mathcal{L}, 1 \leq i \leq n$, then

$$
\psi(x ; n) \sim P\left(\sum_{i=1}^{n} c_{i} X_{i}>x\right) \sim P\left(\sum_{i=1}^{n} c_{i} X_{i}^{+}>x\right) .
$$

If $F_{i} \in \mathcal{D} \cap \mathcal{L}, 1 \leq i \leq n$, then

$$
\psi(x ; n) \sim P\left(\sum_{i=1}^{n} c_{i} X_{i}>x\right) \sim P\left(\sum_{i=1}^{n} c_{i} X_{i}^{+}>x\right) \sim \sum_{i=1}^{n} P\left(c_{i} X_{i}>x\right) .
$$




\section{Proof of Results}

A function $h(x)$ is called slowly varying at infinity if $h(x y) \sim h(x)$ for any $y>0$, It is well-known that $h(x)=o\left(x^{\delta}\right)$ for any $\delta>0$ if $h(x)$ is a slowly varying function, see, e.g., Bingham et al. [2]. The following result is crucial for the proof of all theorems in this paper. It shows that any tail distribution of a long-tailed distribution is uniformly $h$-insensitive for a slowly varying function $h$.

Lemma 5.1. If $X \sim F \in \mathcal{L}$, then $\bar{F}$ is $h$-insensitive for a positive nondecreasing and slowly varying function $h(x):=h(x ; F):(0, \infty) \rightarrow(0, \infty)$ satisfying $h(x) \nearrow \infty, h(x) \leq \operatorname{ch}\left(\frac{x}{c}\right)$ for all $c \geq 1$, and

$$
\lim _{x \rightarrow \infty} \sup _{a(x) \leq c \leq b(x)}\left|\frac{P(c X>x \pm h(x))}{P(c X>x)}-1\right|=0,
$$

where $b(x)$ is an arbitrary positive function such that $b(x) \nearrow \infty$ and $b(x)=o(x)$, and $a(x)=h^{-\delta}(x)$ for some $\delta>0$.

Proof. For any fixed $\delta>0$, let $\left\{x_{n}, n \geq 1\right\}$ be a sequence of increasing positive real numbers such that $x_{n+1} \geq 2 x_{n}>0, n \geq 1$, and for any $x \geq x_{n}$,

$$
\sup _{|y| \leq n}\left|\frac{\bar{F}(x+y)}{\bar{F}(x)}-1\right| \leq \max \left\{\left|\frac{\bar{F}\left(x+n^{1+\delta}\right)}{\bar{F}(x)}-1\right|,\left|\frac{\bar{F}\left(x-n^{1+\delta}\right)}{\bar{F}(x)}-1\right|\right\} \leq \frac{1}{n} .
$$

Borrowing the idea of the proof of Corollary 2.5 in [5], let

$$
h(x)= \begin{cases}\frac{2}{x_{1}} x & x_{0}=0<x<x_{1} \\ n+\frac{x-x_{n-1}}{x_{n}-x_{n-1}} & x_{n-1} \leq x<x_{n}, n \geq 2 .\end{cases}
$$

Clearly, $h(x)$ is a positive nondecreasing, piecewise linear, continuous function and $h(x) \nearrow \infty$. Since $h(x)$ is a nondecreasing function, $h(x y) \sim h(x)$ for any $y>0$ is equivalent to $h(2 x) \sim h(x)$, which follows from the facts that $h(x) \nearrow \infty$ and $h(x) \leq h(2 x)<h\left(x_{n+1}\right)=n+2 \leq h(x)+2$ for any $x_{n-1} \leq x<x_{n}$.

For any $x \geq x_{n}$, i.e., $x \in\left[x_{n+k}, x_{n+k+1}\right)$ for some $k:=k(x) \geq 0$, and $|y| \leq h^{1+\delta}(x)=(n+k+1)^{1+\delta}$, it follows from (8) that

$$
\sup _{|y| \leq h^{1+\delta}(x)}\left|\frac{\bar{F}(x+y)}{\bar{F}(x)}-1\right| \leq \frac{1}{n+k+1} \leq \frac{1}{n} \rightarrow 0, \quad \text { as } n \rightarrow \infty,
$$

i.e., $\bar{F}$ is $h^{1+\delta}$-insensitive, which of course implies that $\bar{F}$ is $h$-insensitive. Since $x_{n+1}-x_{n} \geq x_{n} \geq$ $x_{n}-x_{n-1}, n \geq 1, h^{\prime}(x)$ is a nonincreasing function on $\cup_{n=1}^{\infty}\left(x_{n-1}, x_{n}\right)$, which implies that $h(x)$ is a concave function on $[0, \infty)$. The concavity of $h(x)$ and the fact $h(0)=0$ lead to $h\left(\frac{x}{c}\right)=$ $h\left(\frac{1}{c} x+\left(1-\frac{1}{c}\right) 0\right) \geq \frac{1}{c} h(x)+\left(1-\frac{1}{c}\right) h(0)=\frac{1}{c} h(x)$, i.e., $h(x) \leq c h\left(\frac{x}{c}\right)$, for any $x>0, c>1$.

Hence, $\frac{h(x)}{c} \leq h\left(\frac{x}{c}\right) \leq h^{1+\delta}\left(\frac{x}{c}\right)$ for $1 \leq c \leq b(x)$. Note that $\frac{h(x)}{c} \leq \frac{h(x)}{a(x)}=h^{1+\delta}(x) \leq h^{1+\delta}\left(\frac{x}{c}\right)$ for $a(x) \leq c \leq 1$. The monotonicity of $\bar{F}$ yields $\bar{F}\left(\frac{x}{c}+h^{1+\delta}\left(\frac{x}{c}\right)\right) \leq P(c X>x \pm h(x))=\bar{F}\left(\frac{x}{c} \pm\right.$ $\left.\frac{h(x)}{c}\right) \leq \bar{F}\left(\frac{x}{c}-h^{1+\delta}\left(\frac{x}{c}\right)\right)$ for $a(x) \leq c \leq b(x)$. The uniform asymptotic relation (7) follows from the inequalities

$$
\begin{aligned}
\frac{\bar{F}\left(\frac{x}{c}+h^{1+\delta}\left(\frac{x}{c}\right)\right)}{\bar{F}\left(\frac{x}{c}\right)}-1 & \leq \frac{P(c X>x \pm h(x))}{P(c X>x)}-1=\frac{\left.\bar{F}\left(\frac{x}{c} \pm \frac{h(x)}{c}\right)\right)}{\bar{F}\left(\frac{x}{c}\right)}-1 \\
& \leq \frac{\bar{F}\left(\frac{x}{c}-h^{1+\delta}\left(\frac{x}{c}\right)\right)}{\bar{F}\left(\frac{x}{c}\right)}-1, \quad a(x) \leq c \leq b(x),
\end{aligned}
$$


and the fact that $\bar{F}$ is $h^{1+\delta}$-insensitive.

Remark 5.1. It is easy show that $\frac{h(x)}{x} \searrow 0$ for $h(x)$ in the proof of Lemma 5.1.

Proof of Theorem 3.1. Assume that $\bar{F}_{i}$ is $h_{i}$-insensitive, where $h_{i}(x)=h\left(x ; F_{i}\right)$ is given in Lemma $5.1,1 \leq i \leq n$. Let $h(x):=h\left(x ; F_{1}, \cdots, F_{n}\right)=\min \left\{h_{i}(x), 1 \leq i \leq n\right\}=o(x)$. Then all $\bar{F}_{i}$ 's are $h$-insensitive and $h(x) \leq c h\left(\frac{x}{c}\right), c \geq 1$, by Lemma 5.1. The uniform asymptotic relation (6), which is essentially the case of $n=2$ in proof, will be proved by induction. It is obviously true for $n=1$ by Lemma 5.1. Since distribution functions are nondecreasing, (6) is equivalent to

$$
\lim _{x \rightarrow \infty} \inf _{a(x) \leq c_{i} \leq b(x), 1 \leq i \leq n} \frac{P\left(\sum_{i=1}^{n} c_{i} X_{i}>x+h(x)\right)}{P\left(\sum_{i=1}^{n} c_{i} X_{i}>x\right)} \geq 1,
$$

and

$$
\lim _{x \rightarrow \infty} \sup _{a(x) \leq c_{i} \leq b(x), 1 \leq i \leq n} \frac{P\left(\sum_{i=1}^{n} c_{i} X_{i}>x-h(x)\right)}{P\left(\sum_{i=1}^{n} c_{i} X_{i}>x\right)} \leq 1 .
$$

Write $A+B+C$ for the union of disjoint sets $A, B, C$. The fact that $\left\{\sum_{i=1}^{n} c_{i} X_{i}>x \pm h(x)\right\}=$ $\left\{\sum_{i=1}^{n} c_{i} X_{i}>x+h(x), c_{n} X_{n} \leq \frac{x+h(x)}{2}\right\}+\left\{\sum_{i=1}^{n} c_{i} X_{i}>x+h(x), \sum_{i=1}^{n-1} c_{i} X_{i} \leq \frac{x+h(x)}{2}\right\}+$ $\left\{\sum_{i=1}^{n-1} c_{i} X_{i}>\frac{x+h(x)}{2}, c_{n} X_{n}>\frac{x+h(x)}{2}\right\}$ and independence of $X_{i}$ 's yield

$$
\begin{aligned}
P\left(\sum_{i=1}^{n} c_{i} X_{i}>x+h(x)\right) \geq & \int_{-\infty}^{x / 2} P\left(\sum_{i=1}^{n-1} c_{i} X_{i}>x+h(x)-t\right) d P\left(c_{n} X_{n} \leq t\right) \\
& +\int_{-\infty}^{x / 2} P\left(c_{n} X_{n}>x+h(x)-t\right) d P\left(\sum_{i=1}^{n-1} c_{i} X_{i} \leq t\right) \\
& +P\left(\sum_{i=1}^{n-1} c_{i} X_{i}>\frac{x+h(x)}{2}\right) P\left(c_{n} X_{n}>\frac{x+h(x)}{2}\right) .
\end{aligned}
$$

The induction assumption with $b(x)$ replaced by $2 b(x)$ implies that

$$
\begin{aligned}
& P\left(\sum_{i=1}^{n-1} c_{i} X_{i}>\frac{x+h(x)}{2}\right) P\left(c_{n} X_{n}>\frac{x+h(x)}{2}\right) \\
= & P\left(\sum_{i=1}^{n-1} 2 c_{i} X_{i}>x+h(x)\right) P\left(2 c_{n} X_{n}>x+h(x)\right) \\
\sim & P\left(\sum_{i=1}^{n-1} 2 c_{i} X_{i}>x\right) P\left(2 c_{n} X_{n}>x\right)=P\left(\sum_{i=1}^{n-1} c_{i} X_{i}>\frac{x}{2}\right) P\left(c_{n} X_{n}>\frac{x}{2}\right)
\end{aligned}
$$

holds uniformly for $a(x) \leq c_{i} \leq b(x), 1 \leq i \leq n$.

Use monotonicity of any distribution function and the inequality $h(x) \leq 2 h\left(\frac{x}{2}\right)$ to obtain

$$
1 \geq \inf _{t \leq x / 2} \frac{\bar{F}(x+h(x)-t)}{\bar{F}(x-t)} \geq \inf _{t \leq x / 2} \frac{\bar{F}\left(x-t+2 h\left(\frac{x}{2}\right)\right)}{\bar{F}(x-t)} \geq \inf _{u=x-t \geq x / 2} \frac{\bar{F}(u+2 h(u))}{\bar{F}(u)} \sim 1
$$

provided $\bar{F}$ is $h$-insensitive. It follows from the induction assumption and Lemma 5.1 that the tail distribution of $\sum_{i=1}^{n-1} c_{i} X_{i}$ and the tail distribution of $c_{n} X_{n}$ are $h$-insensitive. The asymptotic 
relation (12) and the inequality (11) imply

$$
\begin{aligned}
& P\left(\sum_{i=1}^{n} c_{i} X_{i}>x+h(x)\right) \\
\geq & \left(\int_{-\infty}^{x / 2} P\left(\sum_{i=1}^{n-1} c_{i} X_{i}>x-t\right) d P\left(c_{n} X_{n} \leq t\right)+\int_{-\infty}^{x / 2} P\left(c_{n} X_{n}>x-t\right) d P\left(\sum_{i=1}^{n-1} c_{i} X_{i} \leq t\right)\right. \\
& \left.\quad+P\left(\sum_{i=1}^{n-1} c_{i} X_{i}>\frac{x}{2}\right) P\left(c_{n} X_{n}>\frac{x}{2}\right)\right)(1+o(1)) \\
= & (1+o(1)) P\left(\sum_{i=1}^{n} c_{i} X_{i}>x\right),
\end{aligned}
$$

where the term $o(1)$ goes to 0 uniformly for $a(x) \leq c_{i} \leq b(x), 1 \leq i \leq n$. This complete the proof of (9).

The other uniform asymptotic relation (10) can be obtained by substituting $+h(x),+2 h\left(\frac{x}{2}\right), \geq$, inf with $-h(x),-2 h\left(\frac{x}{2}\right), \leq$, sup, respectively, in the proof of $(9)$.

Proof of Theorem 3.2. The idea is from the proof of Theorem 2.1 of Chen et al. [3]. Let $\left\{\Omega_{K}=\left\{X_{i} \geq 0\right.\right.$ for all $i \in K, X_{j}<0$ for all $\left.\left.j \in\{1, \cdots, n\} \backslash K\right\}, K \subseteq\{1, \cdots, n\}\right\}$ be a finite partition of the whole space $\Omega$. Obviously, $P\left(\sum_{i=1}^{n} c_{i} X_{i}>x, \Omega_{K}\right)$ is not less than

$$
\begin{aligned}
& P\left(\sum_{i \in K} c_{i} X_{i}>x+h(x), \sum_{j \notin K} c_{j} X_{j}>-h(x), \Omega_{K}\right) \\
= & P\left(\sum_{i=1}^{n} c_{i} X_{i}^{+}>x+h(x), \Omega_{K}\right)-P\left(\sum_{i \in K} c_{i} X_{i}>x+h(x), \sum_{j \notin K} c_{j} X_{j} \leq-h(x), \Omega_{K}\right),
\end{aligned}
$$

where, due to the independence of $X_{i}$ 's, the second term equals

$$
P\left(\sum_{i \in K} c_{i} X_{i}>x+h(x), \bigcap_{i \in K}\left\{X_{i} \geq 0\right\}\right) P\left(\sum_{j \notin K} c_{j}\left(-X_{j}\right) \geq h(x), \bigcap_{j \notin K}\left\{X_{j}<0\right\}\right) .
$$

and it is at most $P\left(\sum_{i=1}^{n} c_{i} X_{i}^{+}>x+h(x)\right) P\left(\sum_{j=1}^{n} c_{j} X_{j}^{-} \geq h(x)\right)$, where $x^{-}=\max \{-x, 0\}$. Note that $\left\{\sum_{j=1}^{n} c_{j} X_{j}^{-} \geq h(x)\right\} \subseteq \bigcup_{j=1}^{n}\left\{c_{j} X_{j}^{-} \geq \frac{h(x)}{n}\right\}=\bigcup_{j=1}^{n}\left\{c_{j} X_{j} \leq-\frac{h(x)}{n}\right\}$, whose probability is at most $\sum_{j=1}^{n} P\left(X_{j} \leq-\frac{h(x)}{n b(x)}\right)=o(1)$ provided $b(x)=o(h(x))$. Therefore, uniformly for $0<a \leq c_{i} \leq$ $b(x), 1 \leq i \leq n$, the second term in (14) is $o\left(P\left(\sum_{i=1}^{n} c_{i} X_{i}^{+}>x+h(x)\right)\right)$ and

$$
P\left(\sum_{i=1}^{n} c_{i} X_{i}>x, \Omega_{K}\right) \geq P\left(\sum_{i=1}^{n} c_{i} X_{i}^{+}>x+h(x), \Omega_{K}\right)+o\left(P\left(\sum_{i=1}^{n} c_{i} X_{i}^{+}>x+h(x)\right)\right) .
$$

Sum it over all $K$ 's to get

$$
P\left(\sum_{i=1}^{n} c_{i} X_{i}>x\right) \geq P\left(\sum_{i=1}^{n} c_{i} X_{i}^{+}>x+h(x)\right)+o\left(P\left(\sum_{i=1}^{n} c_{i} X_{i}^{+}>x+h(x)\right)\right) .
$$

Clearly, $X_{i}^{+} \sim F_{i}^{+}(x)=F_{i}(x) I_{\{x \geq 0\}} \in \mathcal{L}, 1 \leq i \leq n$. Choose $h(x)$ such that (6) holds with $F_{i}$ substituted by $F_{i}^{+}$. The desired result follows from Theorem 3.1 and the simple fact that $\sum_{i=1}^{n} c_{i} X_{i} \leq \max _{1 \leq k \leq n} \sum_{i=1}^{k} c_{i} X_{i} \leq \sum_{i=1}^{n} c_{i} X_{i}^{+}$. 
Proof of Corollary 3.2. Recall that $\bar{F} \in \mathcal{S}$ if $\overline{F^{+}} \in \mathcal{S}$, i.e., $\overline{F^{+} * F^{+}}(x) \sim 2 \overline{F^{+}}(x)$ for $F^{+}(x)=$ $F(x) I_{\{x \geq 0\}}$. Clearly, $F \in \mathcal{L}$ iff $F^{+} \in \mathcal{L}$. If $F^{+} \in \mathcal{S}$, the fact that $\mathcal{S} \subset \mathcal{L}$ implies $F \in \mathcal{L}$. Then it is equivalent to show that $\overline{F^{+} * F^{+}}(x) \sim 2 \overline{F^{+}}(x)$ iff $\overline{F * F}(x) \sim 2 \bar{F}(x)$, i.e. $\overline{F^{+} * F^{+}}(x) \sim \overline{F * F}(x)$ since $\overline{F^{+}}(x)=\bar{F}(x)$ for all $x>0$. It is obviously true by Theorem 3.2.

The next two lemma can be easily checked from the definition of the class $\mathcal{C}$.

Lemma 5.2. If $X$ follows distribution $F \in \mathcal{C}$, then $\bar{F}(x)$ is $h$-insensitive provided $h(x)=o(x)$ and it holds that, uniformly for $0<c<b(x)=o(x)$,

$$
P(c X>x \pm h(x)) \sim P(c X>x) .
$$

Lemma 5.3. If $X_{i} \sim F_{i} \in \mathcal{C}, 1 \leq i \leq n$, are $p Q S A I$ random variables, it holds that, uniformly for $0<c<b(x)=o(x)$,

$$
P\left(c_{j} X_{j}>\frac{x}{n}, \max _{1 \leq k \neq j \leq n}\left|c_{k} X_{k}\right|>b(x) \ln \left(\frac{x}{b(x)}\right)\right)=o\left(P\left(c_{j} X_{j}>x\right)\right)
$$

and consequently

$$
P\left(\bigcup_{j=1}^{n}\left\{c_{j} X_{j}>\frac{x}{n}, \max _{1 \leq k \neq j \leq n}\left|c_{k} X_{k}\right|>b(x) \ln \left(\frac{x}{b(x)}\right)\right\}\right)=o\left(\sum_{j=1}^{n} P\left(c_{j} X_{j}>x\right)\right) .
$$

Proof of Theorem 3.3. Let $h(x)=b(x) \ln \left(\frac{x}{b(x)}\right)$. The proof is similar to that of Theorem 3.4 and is omitted.

Proof of Corollary 3.3. Partition the range of the weights as $\left\{\left(c_{1}, \cdots, c_{n}\right): 0 \leq c_{i} \leq b(x), 1 \leq\right.$ $\left.i \leq n, \min _{i=1}^{n} c_{i}>0\right\}=\bigcup_{K \subset\{1, \ldots, n\}}\left\{\left(c_{1}, \cdots, c_{n}\right): 0 \leq c_{i} \leq b(x), i \in K, 0<c_{i} \leq b(x), i \notin K\right\}$. The desired result follows from Theorem 3.3.

Lemma 5.4. If $X_{i} \sim F_{i} \in \mathcal{D}, 1 \leq i \leq n$, are $p S Q A I$ random variables, $h(x)=o(x)$ and $h(x) \nearrow \infty$, it holds that, uniformly for $0<a<c_{i}<b(x)=o(h(x)), 1 \leq i \leq n$,

$$
P\left(c_{j} X_{j}>\frac{x}{n}, \max _{1 \leq k \neq j \leq n}\left|c_{k} X_{k}\right|>h(x)\right)=o\left(P\left(c_{j} X_{j}>x\right)\right)
$$

and consequently

$$
P\left(\bigcup_{j=1}^{n}\left\{c_{j} X_{j}>\frac{x}{n}, \max _{1 \leq k \neq j \leq n}\left|c_{k} X_{k}\right|>h(x)\right\}\right)=o\left(\sum_{j=1}^{n} P\left(c_{j} X_{j}>x\right)\right) .
$$

Proof. The results follow from the fact that $F_{i} \in \mathcal{D}$ and $b(x)=o(h(x))$, the pSQAI property of $X_{i}$ 's and the elementary probability inequality $P\left(A \cap \cup_{i=1}^{n} B_{i}\right) \leq \sum_{i=1}^{n} P\left(A B_{i}\right)$.

If $X_{i}$ is large, the pSQAI property of $X_{j}$ 's implies that other $X_{j}$ 's are relatively close to 0 and negligible compared with $X_{i}$. If $\sum_{i=1}^{n} c_{i} X_{i}>x$, there should be exactly one $c_{i} X_{i}$ greater than $\frac{x}{n}$ and consequently Lemma 5.4 implies

$$
P\left(\sum_{i=1}^{n} c_{i} X_{i}>x\right) \sim \sum_{j=1}^{n} P\left(\sum_{i=1}^{n} c_{i} X_{i}>x, c_{j} X_{j}>\frac{x}{n}, \max _{1 \leq k \neq j \leq n}\left|c_{k} X_{k}\right| \leq h(x)\right) .
$$

It gives the idea of the proof of Theorem 3.4, which is simpler and more straightforward than the proof of Lemma 2.1 of Liu et al. [16] and Theorem 2.1 of Li [14]. 
Proof of Theorem 3.4. All asymptotic relations hold uniformly for $a(x) \leq c_{i} \leq b(x), 1 \leq i \leq n$, in the proof. By Lemma 5.1, there exists a positive nondecreasing function $h(x):=h\left(x, a ; F_{1}, \cdots, F_{n}\right)$ satisfying $h(x) \nearrow \infty$ and $h(x)=o(x)$ such that (7) holds for $F=F_{i}, 1 \leq i \leq n$, respectively. Choose $b(x)=o(h(x))$ and $b(x) \nearrow \infty$. Note that

$$
\begin{aligned}
\left\{\sum_{i=1}^{n} c_{i} X_{i}>x\right\} & =\bigcup_{j=1}^{n}\left\{\sum_{i=1}^{n} c_{i} X_{i}>x, c_{j} X_{j}>\frac{x}{n}\right\} \\
& =\bigcup_{j=1}^{n} A_{j} \bigcup\left\{\sum_{i=1}^{n} c_{i} X_{i}>x, \bigcup_{j=1}^{n}\left\{c_{j} X_{j}>\frac{x}{n}, \max _{1 \leq k \neq j \leq n}\left|c_{k} X_{k}\right|>h(x)\right\}\right\},
\end{aligned}
$$

where $A_{j}=\left\{\sum_{i=1}^{n} c_{i} X_{i}>x, c_{j} X_{j}>\frac{x}{n}, \max _{1 \leq k \neq j \leq n}\left|c_{k} X_{k}\right| \leq h(x)\right\}, 1 \leq j \leq n$, are mutually exclusive events provided $\frac{x}{n}>h(x)$. The elementary probability inequality $P(A) \leq P(A \cup B) \leq$ $P(A)+P(B)$ and Lemma 5.4 lead to

$$
P\left(\sum_{i=1}^{n} c_{i} X_{i}>x\right)=\sum_{j=1}^{n} P\left(A_{j}\right)+o\left(\sum_{j=1}^{n} P\left(c_{j} X_{j}>x\right)\right) .
$$

Lemma 5.1 and the fact that $c_{j} X_{j}$ is at least $x-(n-1) h(x)$ on $A_{j}$ lead to

$$
P\left(A_{j}\right) \leq P\left(c_{j} X_{j}>x-(n-1) h(x)\right)=P\left(c_{j} X_{j}>x\right)+o\left(P\left(c_{j} X_{j}>x\right)\right), \quad 1 \leq j \leq n .
$$

Since $\max _{1 \leq k \neq j \leq n}\left|c_{k} X_{k}\right| \leq h(x)$ on $A_{j}, c_{j} X_{j}>x+(n-1) h(x)$ implies $\sum_{i=1}^{n} c_{i} X_{i}>x$ on $A_{j}$ for any $1 \leq j \leq n$. It follows from Lemma 5.1 and 5.4 that

$$
\begin{aligned}
P\left(A_{j}\right) & \geq P\left(c_{j} X_{j}>x+(n-1) h(x), \max _{1 \leq k \neq j \leq n}\left|c_{k} X_{k}\right| \leq h(x)\right) \\
& =P\left(c_{j} X_{j}>x+(n-1) h(x)\right)-P\left(c_{j} X_{j}>x+(n-1) h(x), \max _{1 \leq k \neq j \leq n}\left|c_{k} X_{k}\right|>h(x)\right) \\
& =P\left(c_{j} X_{j}>x\right)+o\left(P\left(c_{j} X_{j}>x\right)\right), \quad 1 \leq j \leq n .
\end{aligned}
$$

Therefore, (15) can be written as

$$
P\left(\sum_{i=1}^{n} c_{i} X_{i}>x\right) \sim \sum_{i=1}^{n} P\left(c_{i} X_{i}>x\right) .
$$

In the exactly same way, it can be proved that

$$
P\left(\sum_{i=1}^{n} c_{i} X_{i}^{+}>x\right) \sim \sum_{i=1}^{n} P\left(c_{i} X_{i}^{+}>x\right)=\sum_{i=1}^{n} P\left(c_{i} X_{i}>x\right) .
$$

Note that $\sum_{i=1}^{n} c_{i} X_{i} \leq \max _{1 \leq k \leq n} \sum_{i=1}^{k} c_{i} X_{i} \leq \sum_{i=1}^{n} c_{i} X_{i}^{+}$. The desired results follow from the uniform asymptotic relation (16) and (17).

Remark 5.2. The proof of Theorem 3.4 also leads to Corollary 3.4.

\section{Acknowledgments}

The author would like to thank the anonymous referees for their comments and help in improving the paper. 


\section{References}

[1] Barbe, P. and McCormick, W.P. (2009). Asymptotic expansions for infinite weighted convolutions of heavy tail distributions and applications. Memoirs of the American Mathematical Society. 197, $1-117$.

[2] Bingham, N.H., Goldie, C.M., Teugels, J.L. (1989) Regular Variation. Cambridge University Press, Cambridge.

[3] Chen, Y., Ng, K.W., Yuen, K.C. (2011) The maximum of randomly weighted sums with long tails in insurance and finance. Stoch. Anal. Appl. 29, 1033-1044.

[4] Chen, Y., Yuen, K.C. (2009) Sums of pairwise quasi-asymptotically independent random variables with consistent variation. Stoch. Models 25, 76-89.

[5] Cline, D.B.H., Samorodnitsky, G. (1994) Subexponentiality of the product of independent random variables. Stoch. Process. Their. Appl. 49, 75-98.

[6] Denisov, D., Foss, S., Korshunov, D. (2010) Asymptotics of randomly stopped sums in the presence of heavy tails. Bernoulli 16, 971-994.

[7] Embrechts, P., Goldie, C. (1980) On closure and factorization properties of subexponential and related distributions. J. Austral. Math. Soc. 29, 243-256.

[8] Embrechts, P., Klüppelberg, C., Mikosch, T. (1997) Modelling extremal events for insurance and finance. Springer. Berlin.

[9] Foss, S., Korshunov, D., Zachary, S. (2011) An introduction to heavy-tailed and subexponential distributions. Springer. New York.

[10] Foss, S., Zachary, S. (2003) The maximum on a random time interval of a random walk with long-tailed increments and negative drift. Ann. Appl. Probab. 13, 37-53.

[11] Geluk, J., Ng, K.W. (2006) Tail behavior of negatively associated heavy-tailed sums. J. Appl. Probab. 43(2), 587-593.

[12] Geluk, J., Tang, Q. (2009) Asymptotic tail probabilities of sums of dependent subexponential random variables. J. Theoret. Prob. 22, 871-882.

[13] Klüppelberg, C. (1988) Subexponential distributions and integrated tails. J. Appl. Probab. 25, $132-141$.

[14] Li, J. (2013) On pairwise quasi-asymptotically independent random variables and their applications. Statist. Probab. Lett. 83, 2081-2087.

[15] Li, J., Tang, Q., Wu, R. (2010). Subexponential tails of discounted aggregate claims in a timedependent renewal risk model. Adv. Appl. Probab. 42, 1126-1146.

[16] Liu, X., Gao, Q., Wang, Y. (2012) A note on a dependent risk model with constant interest rate. Statist. Probab. Lett. 82, 707-712. 
[17] Resnick, S.I. (2002) Hidden regular variation, second order regular variation and asymptotic independence. Extremes 5, 303-336.

[18] Ng, K.W., Tang, Q., Yang, H. (2002), Maxima of sums of heavy-tailed random variables. Astin Bull. 32, 43-55.

[19] Tang, Q. (2004) The ruin probability of a discrete time risk model under constant interest rate with heavy tails. Scand. Actuarial J. 3, 229-240.

[20] Tang, Q. (2008) Insensitivity to negative dependence of asymptotic tail probabilities of sums and maxima of sums. Stoch. Anal. Appl. 26, 435-450.

[21] Tang, Q., Tsitsiashvili, G. (2003) Randomly weighted sums of subexponential random variables with application to ruin theory. Extremes 6, 171-188.

[22] Wang D., Tang Q. (2004) Maxima of sums and random sums for negatively associated random variables with heavy tails. Statist. Probab. Lett. 68, 287-295.

[23] Wang, D., Tang, Q. (2006) Tail probabilities of randomly weighted sums of random variables with dominated variation. Stoch. Models 22, 253-272.

[24] Zhang, Y., Shen, X., Weng, C. (2009) Approximation of the tail probability of randomly weighted sums and applications. Stoch. Proc. Appl. 119, 655-675. 